\title{
Project 8: Towards a Direct Measurement of the Neutrino Mass with Tritium Beta Decays
}

\author{
N. S. Oblath ${ }^{* \dagger}$ \\ Pacific Northwest National Laboratory, Richland, WA, USA \\ E-mail: noah.oblath@pnnl.gov
}

\begin{abstract}
Cyclotron Radiation Emission Spectroscopy, a frequency-based method for determining the energy of relativistic electrons, has recently been demonstrated by the Project 8 collaboration. Applying this technique to the tritium endpoint provides a new avenue for measuring the absolute mass-scale of the neutrino. The proof of principle was done in a small waveguide detector using gaseous ${ }^{83 \mathrm{~m}} \mathrm{Kr}$ as a source of monoenergetic electrons. As the next step towards a neutrino mass measurement, we are upgrading the existing detector to operate using a molecular tritium source and to have enhanced radiofrequency properties. These upgrades are the next research and development steps needed to design a larger scale experiment that will approach the existing neutrino mass limits. I will discuss the expected physics reach of this second phase of Project 8 with molecular tritium, based on data from its commissioning with ${ }^{83 \mathrm{~m}} \mathrm{Kr}$. I will also present the concept for Phases III and IV, which will have a neutrino mass sensitivity similar to current limits from the Mainz and Troitsk experiments.
\end{abstract}

XVII International Workshop on Neutrino Telescopes

13-17 March 2017

Venezia, Italy

${ }^{*}$ Speaker.
${ }^{\dagger}$ On behalf of the Project 8 Collaboration 


\section{Neutrino Mass via Tritium Beta Decay}

While neutrino oscillation experiments have successfully shown that neutrinos change flavor, and therefore have non-zero mass, the absolute mass scale remains unknown. The simplest way to directly measure the mass of the neutrino is using beta decays. Neutrino mass has an effect on the kinematics of the decay process [1]. While the neutrinos themselves are difficult to measure, the energies of the outgoing electrons can be precisely determined. The neutrino mass can then be inferred from the shape of the electron energy spectrum:

$$
\frac{d N}{d K_{e}} \propto F\left(Z, K_{e}\right) \cdot p_{e} \cdot\left(K_{e}+m_{e}\right) \cdot\left(E_{0}-K_{e}\right) \cdot \sum_{i=1}^{3}\left|U_{e i}\right|^{2} \sqrt{\left(E_{0}-K_{e}\right)^{2}-m_{i}^{2}} \cdot \Theta\left(E_{0}-K_{e}-m_{i}\right) .
$$

The Fermi function, $F\left(Z, K_{e}\right)$, takes into account the Coulomb interactions of the electron with the recoiling nucleus; $Z$ is the proton number of the final-state nucleus, $K_{e}$ is the electron's kinetic energy, $p_{e}$ is the electron's momentum, $E_{0}$ is the Q-value of the decay, and $U_{e i}$ are the elements of the PMNS matrix for neutrino mass states $m_{i, i=1-3}$. The only dependence on the neutrino mass comes from the phase-space factor. The shape of the spectrum is independent of all other properties of the neutrino, including whether neutrinos are Majorana or Dirac particles.

One technique being used to precisely measure the beta-decay spectrum relies on a spectrometer to precisely select high-energy electrons from tritium decays. The most recent experiments to use this technique are the Mainz [2] and Troitsk [3] experiments. They placed similar limits on the neutrino mass: $m_{\beta v}<2 \mathrm{eV}$ [4]. KATRIN, the next-generation of spectrometer-type experiments, aims to lower that limit by an order of magnitude, to $200 \mathrm{meV}$ (90\% CL) [5]. KATRIN is currently undergoing commissioning in Karlsruhe, Germany.

The lower limits for the neutrino mass from oscillation experiments $(\approx 10 \mathrm{meV})$ provide a strong motivation for probing to lower neutrino masses. However, with KATRIN, the technologies used in spectrometer-type tritium experiments have been pushed to their current practical limits. A new technique is needed to push the mass sensitivity lower.

\section{A New Technique}

The Project 8 collaboration proposes an alternate method of measuring the electron energies that was originally proposed in [6]: measure the cyclotron radiation emitted by the electrons spiraling around magnetic field lines. This technique is called Cyclotron Radiation Emission Spectroscopy, or CRES. An enclosed volume of tritium is placed in a uniform magnetic field, and as the tritium nuclei decay, the electrons will spiral around the magnetic field lines. The spiraling electrons are being accelerated, and therefore emit cyclotron radiation. The frequency of that radiation is proportional to the magnetic field strength, and inversely proportional to the electron's kinetic energy:

$$
\omega_{\gamma}=\frac{e B}{\gamma m_{e}}=\frac{\omega_{c}}{\gamma} \approx \frac{\omega_{c}}{1+K_{e} /\left(m_{e} c^{2}\right)} .
$$

By measuring the frequency of the cyclotron radiation, one can measure the electron's kinetic energy without interfering with the electron itself. The pitch angle, $\theta$, is the angle between the 
electron momentum vector and the magnetic field. Using a 1-T magnetic field, the endpoint of the tritium spectrum $(18.6 \mathrm{keV})$ falls around $26 \mathrm{GHz}$. The power emitted as cyclotron radiation depends both on the relativistic velocity of the electron, $\beta$, and $\theta$ :

$$
P(\beta, \theta)=\frac{1}{4 \pi \varepsilon_{0}} \frac{2 q^{2} \omega_{c}^{2}}{3 c} \frac{\beta^{2} \sin ^{2} \theta}{1-\beta^{2}} .
$$

The electrons that radiate the most power will be the easiest to detect. Equation 2.2 shows that the power will be greatest for electrons with $\theta \approx 90^{\circ}$. Conveniently, these electrons also travel the slowest in the direction of the magnetic field, increasing the amount of time they can be observed.

The primary concern for making a precise electron energy measurement is the ability to measure frequency precisely. The desired energy precision is therefore the place to start in considering the requirements for this type of experiment. To achieve the necessary energy precision, $\Delta E$, we need to achieve a relative frequency precision of $\Delta f / f=\Delta E / m_{e}$. KATRIN is designed to achieve $\Delta E \approx 1 \mathrm{eV}$; for Project 8 to achieve a similar accuracy means that $\Delta f / f \approx 2 \times 10^{-6}$. This accuracy is reasonable with current technologies. With a 1-T magnetic field, $\Delta f \approx 52 \mathrm{kHz}$ at $26 \mathrm{GHz}$.

The desired frequency accuracy determines for how long we must be able to observe single electrons. To have a frequency resolution of $\Delta f$, we must measure each electron for $t_{\min }=1 / \Delta f$. With the design parameters discussed above, the electrons must be coherently measured for at least $20 \mu \mathrm{s}$. The minimum measurement time places constraints on a number of physical parameters of the experiment. The gas density must be low enough that, on average, $18.6 \mathrm{keV}$ electrons can travel for $t_{\min }$ without scattering. Furthermore, the experiment must be large enough so that the electron can be tracked continuously.

The signal detected for a single electron can be more complicated than the single frequency at which the cyclotron radiation is emitted. In particular, the detected signal can include a Doppler shift due to the velocity of the electron parallel to the magnetic field, a dependence on the electronantenna distance, and effects from the angular dependence of the power distribution of the radiation.

The Project 8 Collaboration is taking a phased approach to developing the technology necessary to turn these cyclotron-radiation signals into a leading-edge neutrino-mass experiment. Phase I was intended to demonstrate that the single-electron cyclotron radiation could be measured, and that those measurements could be used to produce an electron energy spectrum. Phase II, the current phase of operations, is aimed at making the first CRES measurement with a molecular tritium source. Phase III will demonstrate that the technique is physically scalable to sizes that are capable of placing a limit on the neutrino mass competitive with the current limits [4]. Phase IV is the final planned phase of the experiment, in which we will be able to measure the neutrino mass as low as $40 \mathrm{meV}$.

\section{Phase I: CRES Demonstrator}

\subsection{Apparatus}

In the years leading up to 2014 the Project 8 collaboration constructed an apparaturs to demonstrate the CRES technique. The initial goals of the Phase I experiment were to verify that we could detect the cyclotron radiation from a single electron, and then to use such detections to perform a spectroscopic measurement. We used a ${ }^{83 \mathrm{~m}} \mathrm{Kr}$ radioactive source, which emits conversion electrons 
with energies of 7, 9, 17.8, 30, and $32 \mathrm{keV}$ and has a half-life of 1.83 hours. The source is a good stand-in for tritium: it is gaseous, emitting the electrons isotropically, and the energy of one of the electron lines is close to the tritium-decay endpoint of $18.6 \mathrm{keV}$.

Figure 1 shows a diagram of the magnet insert for the experiment, which was located at the University of Washington, in Seattle, WA, USA. A superconducting solenoid provided the 1-T magnetic field. The electrons were trapped in a small $\left(\approx 200 \mathrm{~mm}^{3}\right)$ magnetic bottle in the bore of the magnet. The magnetic field from the solenoid trapped the electrons in the horizontal plane; a trapping coil within the bore of the magnet decreased the field slightly in a small volume, trapping the electrons vertically. Whether or not electrons were trapped depended on the depth of the magnetic bottle potential, and the pitch angle of the electrons. Electrons with large pitch angles $\left(\theta \geq 85^{\circ}\right)$ would be trapped. Fortunately, these electrons also emitted the most power as cyclotron radiation. Though this angle selection severely limited the number of electrons we detected, it maximized the signal-to-noise ratio for each electron.
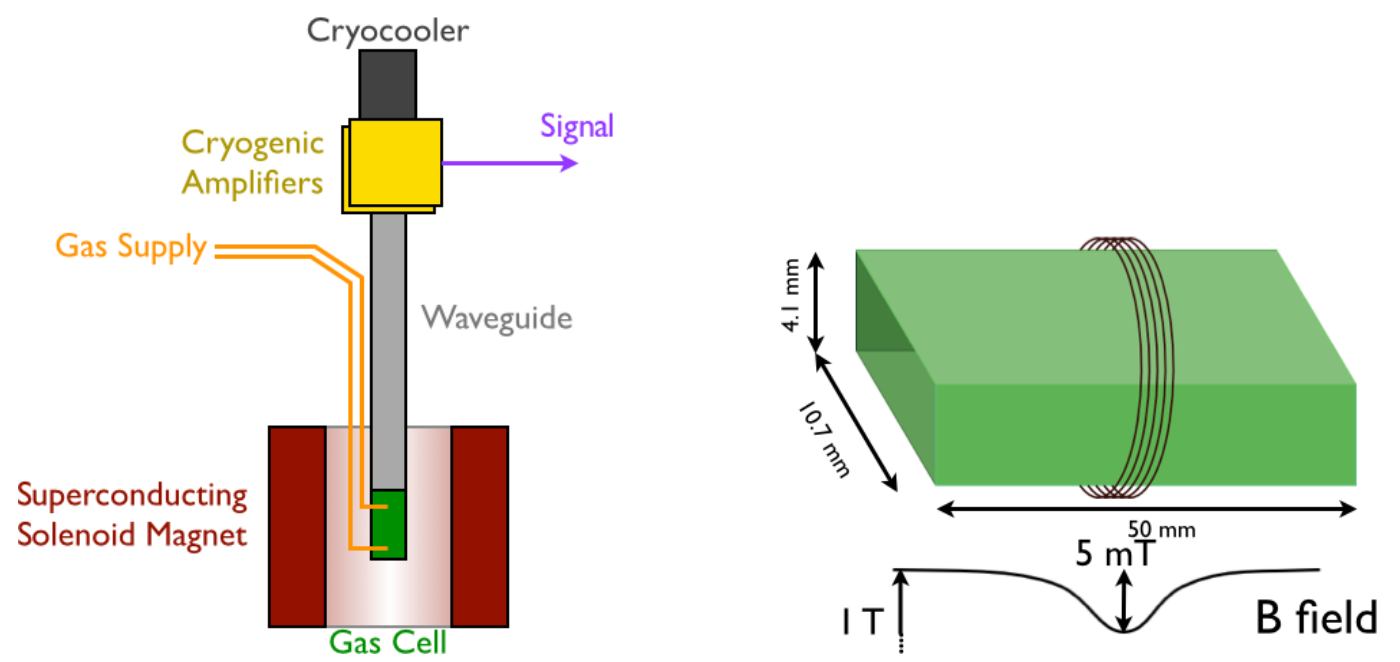

Figure 1: (left) Schematic diagram of the Phase I experiment. (right) Diagram of the gas cell, rotated $90^{\circ}$ with respect to the diagram at left, that is inserted in the solenoid magnet. The gas cell comprised a $5 \mathrm{~cm}$ length of WR-42 aluminum waveguide stock. A magnet coil wound around the gas cell provided a small dip in the magnetic field to trap electrons for observation.

The cyclotron radiation coupled to the $\mathrm{TE}_{10}$ mode of the WR-42 waveguide, and was then amplified by two low-noise cryogenic amplifiers. The rectangular cavity of the waveguide also served as the gas cell to contain the ${ }^{83 \mathrm{~m}} \mathrm{Kr}$ gas. Signals from the amplifiers were mixed down to baseband, digitized and written to disk. After the data were recorded, we analyzed it to search for excesses of power as a function of frequency.

\subsection{First Observation and Spectroscopy}

Figure 2 shows the first event in our data, collected in the first second of data recorded in 2014. We demonstrated that these events were electrons from ${ }^{83 \mathrm{~m}} \mathrm{Kr}$ decays by showing that they only appeared in the frequency bands we expected for 17.8-, 30-, and 32-keV electrons (7- and 9-keV electrons were initially too low in power to be detected), and that we did not see any if 
we reversed the polarity of our magnetic trap. This first observation of single-electron cyclotron radiation was published in [7].

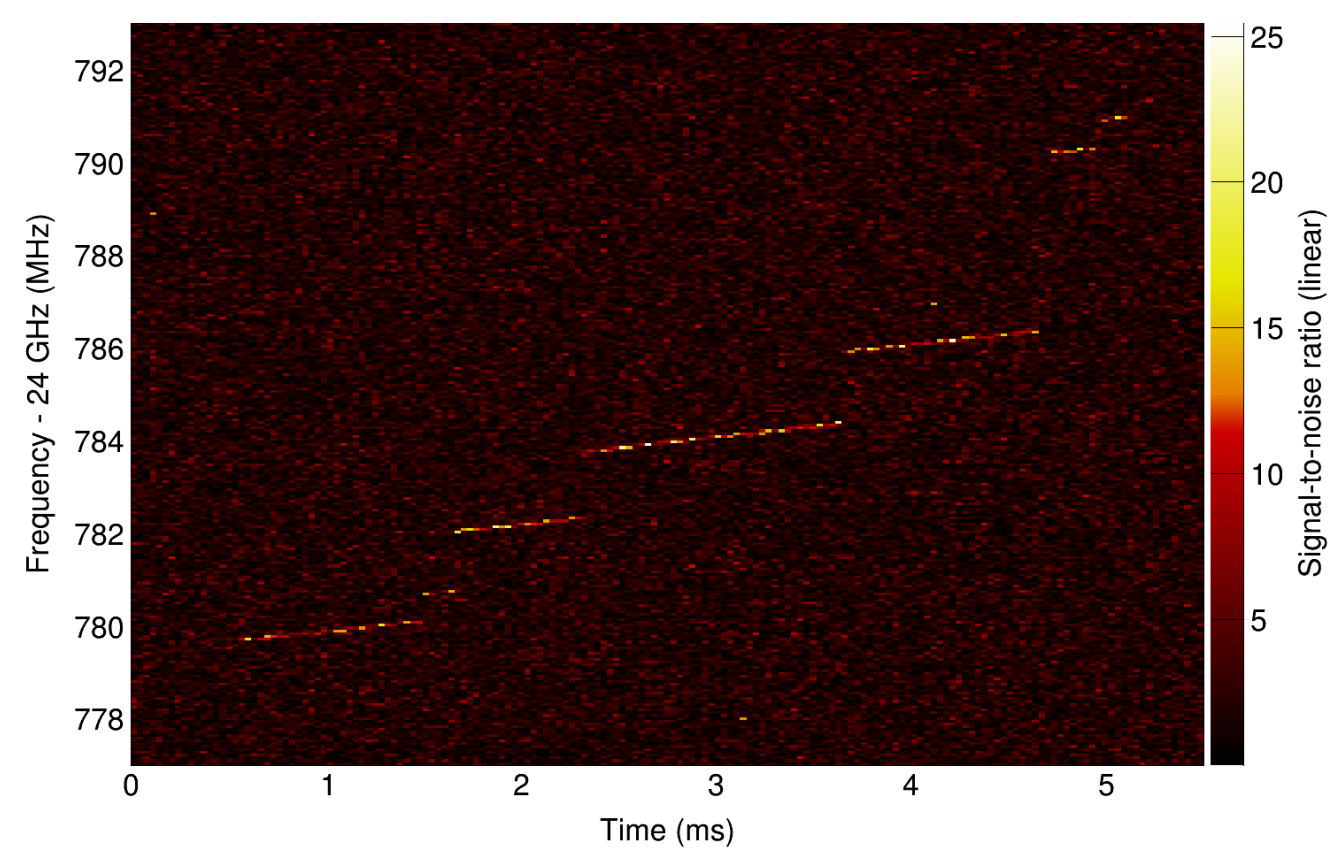

Figure 2: A spectrogram of the first electron detected with the Phase I apparatus. All of the tracks seen were created by a single electron as it scattered off of gas molecules in the cell. The slope of each track is due to energy loss to cyclotron radiation. Eventually the electron scatters in a way that it is no longer trapped by the magnetic fields.

The spectrogram in Fig. 2 shows several important features that were observed for most events. Perhaps the most obvious feature is that there are multiple "tracks" that follow each other immediately in time, but have significant jumps in frequency. Each set of tracks with one immediately following the other in time are from a single electron that scatters off of gas molecules (hydrogen was the dominant residual gas) and ends up at a new frequency because the energy and pitch-angle change. The onset of trapping is clearly visible as the start of the first line on the left side of the spectrogram. Eventually the electron scatters into a state that is no longer trapped by the magnetic fields, and the tracks in the spectrogram stop.

Another striking feature is that the tracks all slope upwards in frequency as a function of time. The rise in frequency of a single track is due to the electron losing energy to cyclotron radiation as it travels in the magnetic field.

Though each track slopes upwards, and in this spectrogram all of the jumps between tracks go upwards in frequency, there are many examples in our dataset where the jumps between tracks go down in frequency as well. The change in frequency between tracks depends both on the change in energy of the electron when it scatters, and the change in pitch angle. Though the former always results in an electron with lower energy (and therefore higher frequency), the latter can cause the frequency to go up or down. 
After identifying individual electron events, the cyclotron frequency of each was converted to an energy using Eq. 2.1 (under the approximation that $\theta=90^{\circ}$ ), and we can produce a spectrum of the ${ }^{83 \mathrm{~m}} \mathrm{Kr}$ conversion electrons. Figure 3 shows the energy spectrum of ${ }^{83 \mathrm{~m}} \mathrm{Kr}$, as reported in [7]. The background spectrum shows the wide view of the 17.8-, 30-, and 32-keV electrons, while the inset zooms in on the set of peaks around $30 \mathrm{keV}$. The two spectra have different resolutions because the depth of the magnetic trap was different when collecting the two datasets. For the background plot the trap was approximately $3.3 \mathrm{mT}$ deep, and the energy resolution was approximately $100 \mathrm{eV}$. For the inset spectrum, the trap was approximately $1.6 \mathrm{mT}$ deep, and the energy resolution improved to $10 \mathrm{eV}$. In a shallower trap the magnetic field is more uniform over the volume of the trap, resulting in an improved energy resolution.

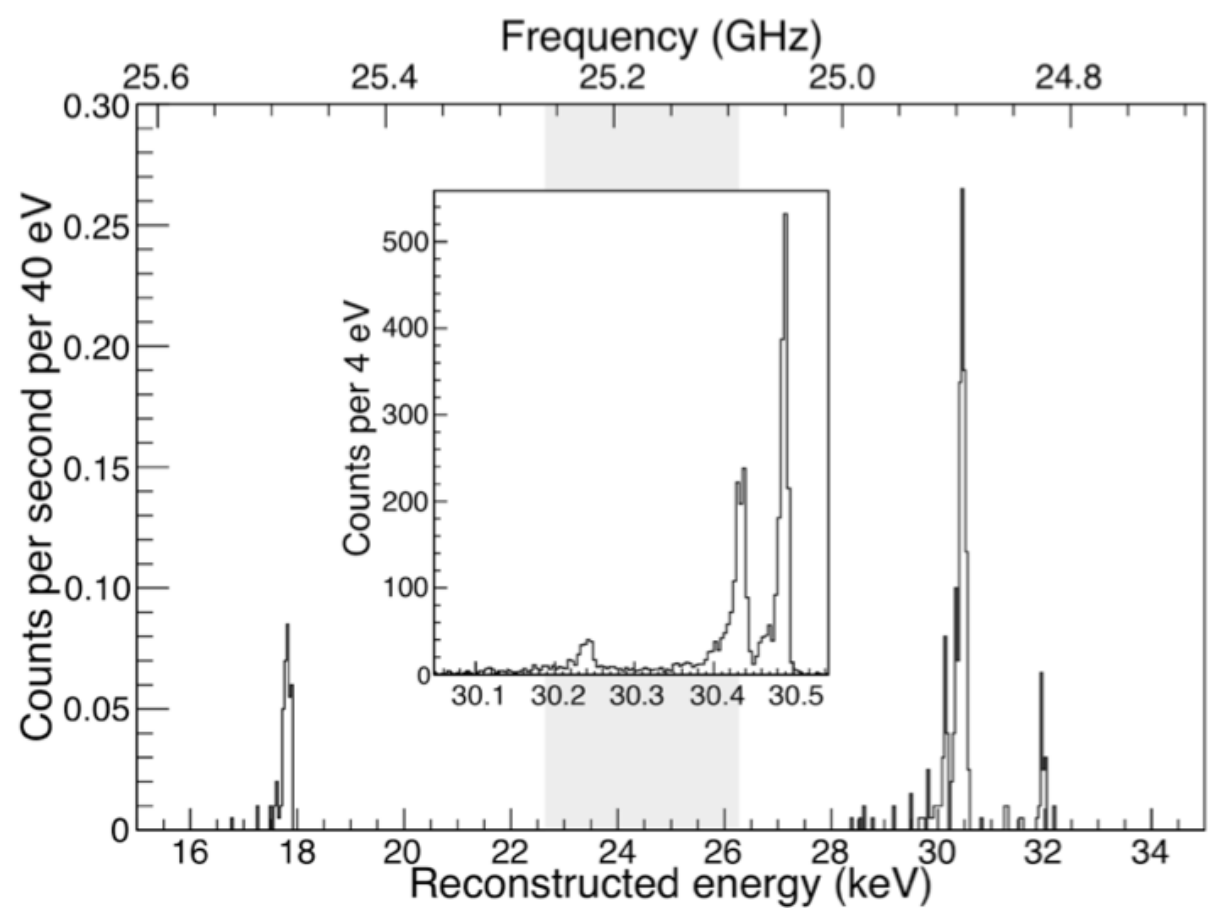

Figure 3: (background) The full ${ }^{83 \mathrm{~m}} \mathrm{Kr}$ spectrum, including the $17.8 \mathrm{keV}, 30 \mathrm{keV}$, and $32 \mathrm{keV}$ electron peaks. The trap depth for this dataset was $\approx 3.3 \mathrm{mT}$, and the energy resolution was $\approx 100 \mathrm{eV}$. (inset) The spectrum of the peaks around $30 \mathrm{keV}$, measured with a trap depth of $\approx 1.6 \mathrm{mT}$, and an energy resolution of $\approx 10 \mathrm{eV}$. The separation between the $30-\mathrm{keV}$ doublet is $52.8 \mathrm{eV}$.

\subsection{Improving the Energy Resolution}

We have two main methods of seeking to improve the energy resolution of the spectrometer: change the trap geometry, and remove the dependence of the frequency on pitch angle. For the former, we used an alternate trap geometry called a "bathtub" trap, as is shown in Fig. 4. Two magnet coils were used, with their currents reversed relative to the current direction used in the single-coil trap mode. This produces two bumps in the magnetic field, with a flatter region in the middle where the electrons are trapped. There was therefore a larger volume at the center of the trap with a more uniform field, which lead to an improved energy resolution. It had the additional benefit of significantly increasing the volume of the trapping region, which produced a greater 
number of electron detections. In [8] we reported the first ${ }^{83 \mathrm{~m}} \mathrm{Kr}$ measurements with a bathtub trap configuration. The spectra for the $30 \mathrm{keV}$ and $32 \mathrm{keV}$ electrons are shown in Fig. 5, where we measured FWHM resolutions of $3.3 \mathrm{eV}$ and $3.6 \mathrm{eV}$, respectively.

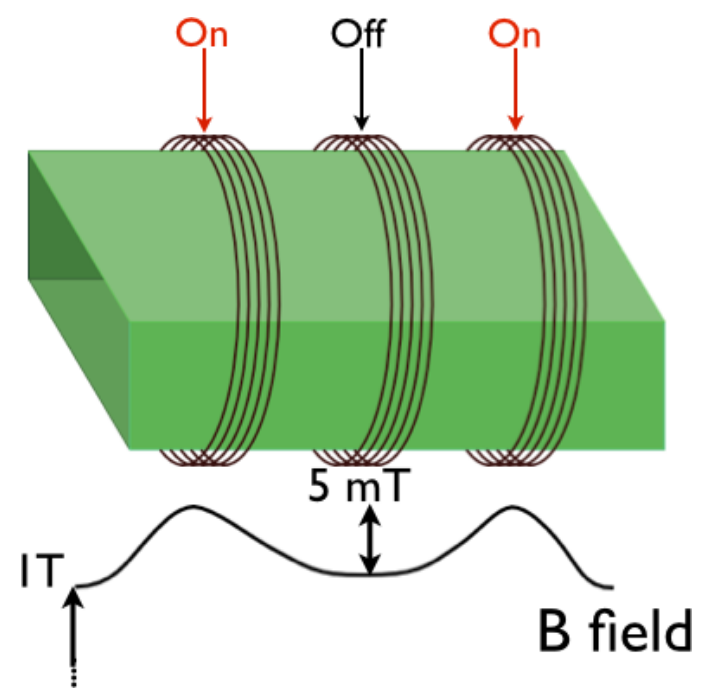

Figure 4: Diagram of the gas cell using the "bathtub" trap setup. The two side coils are energized, and the center coil is turned off.
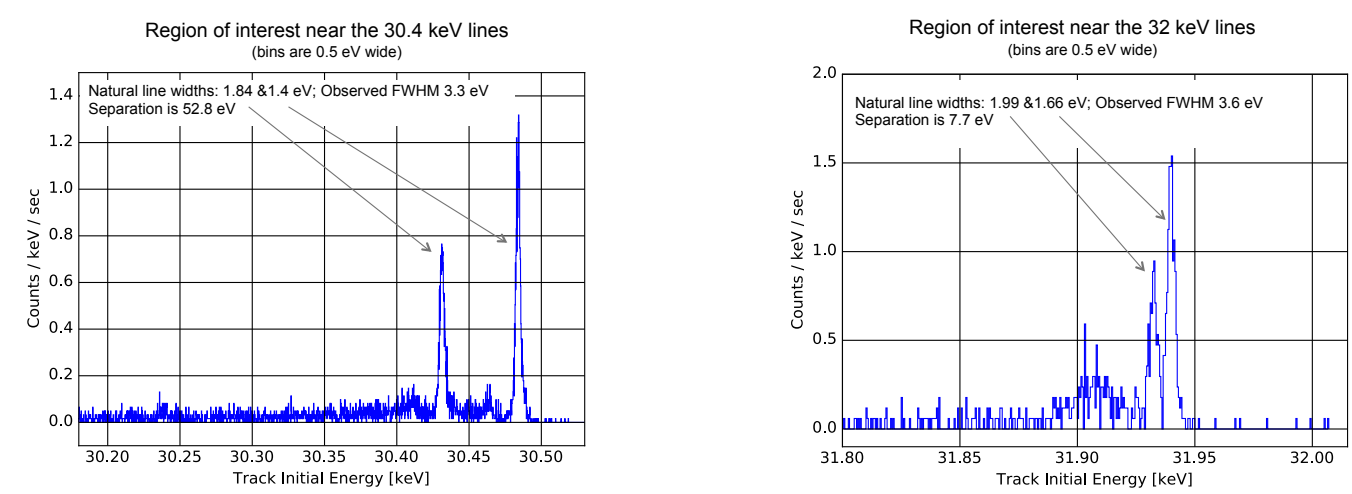

Figure 5: Energy spectra for the $30 \mathrm{keV}$ (left) and $32 \mathrm{keV}$ (right) electrons in a "bathtub" trap configuration.

The second method for improving the resolution of our experiment, which is applicable to all phases, is to separate the dependency of the cyclotron radiation on the electron energy from the dependency on the pitch angle. If the only frequency observed is $\omega_{\gamma}$ from Eq. 2.1, the conversion of frequency to energy will always have an extra uncertainty due to the potential variation in pitch angle. However, the axial frequency of the electron, the frequency with which it travels back and forth in the trap in the direction of the magnetic field, depends strongly on the pitch angle. For example, for a certain approximation of the bathtub trap, the axial frequency is:

$$
\omega_{a} \propto v\left(\frac{a}{\sin \theta}+\frac{4 \sin \theta}{m \cos ^{2} \theta}\right)^{-1}
$$


where $a$ and $m$ are characteristics of the trap shape, and $v$ is the speed of the electron. Due to the Doppler effect on the cyclotron radiation, some of the power emitted leaks from the main frequency peak into sidebands separated by factors of $\omega_{a}$ from $\omega_{\gamma}$. We observed sidebands in data taken with the Phase I apparatus operating in the bathtub-trap mode. Fig. 6 shows a spectrogram with a central peak starting around $92 \mathrm{MHz}$, and a pair of sidebands separated by approximately $40 \mathrm{MHz}$ on either side.

With the first observations of sidebands we made a further discovery: many of the sidebands, including those in Fig. 6, oscillate in frequency at roughly $10 \mathrm{kHz}$. The hypothesis to explain this phenomenon, which is backed up by simulations, is that the trapping coils are tilted very slightly due to the inevitable imperfections in how they were wound, introducing a small asymmetry in shape of the magnetic bottle that traps the electrons: in the cross-sectional plane of the waveguide, on one side the trap will be slightly shorter than on the other side. Electrons move in a wide circle in that plane due to magnetron motion, so they experience the change in trap shape. That change directly affects the axial frequency. Though this effect was not predicted in advance, it serendipitously may allow us to differentiate sideband tracks from central peaks, improving the reliability of our event reconstruction. We are currently developing the analysis tools to take advantage of the information that can be gained from the sidebands.

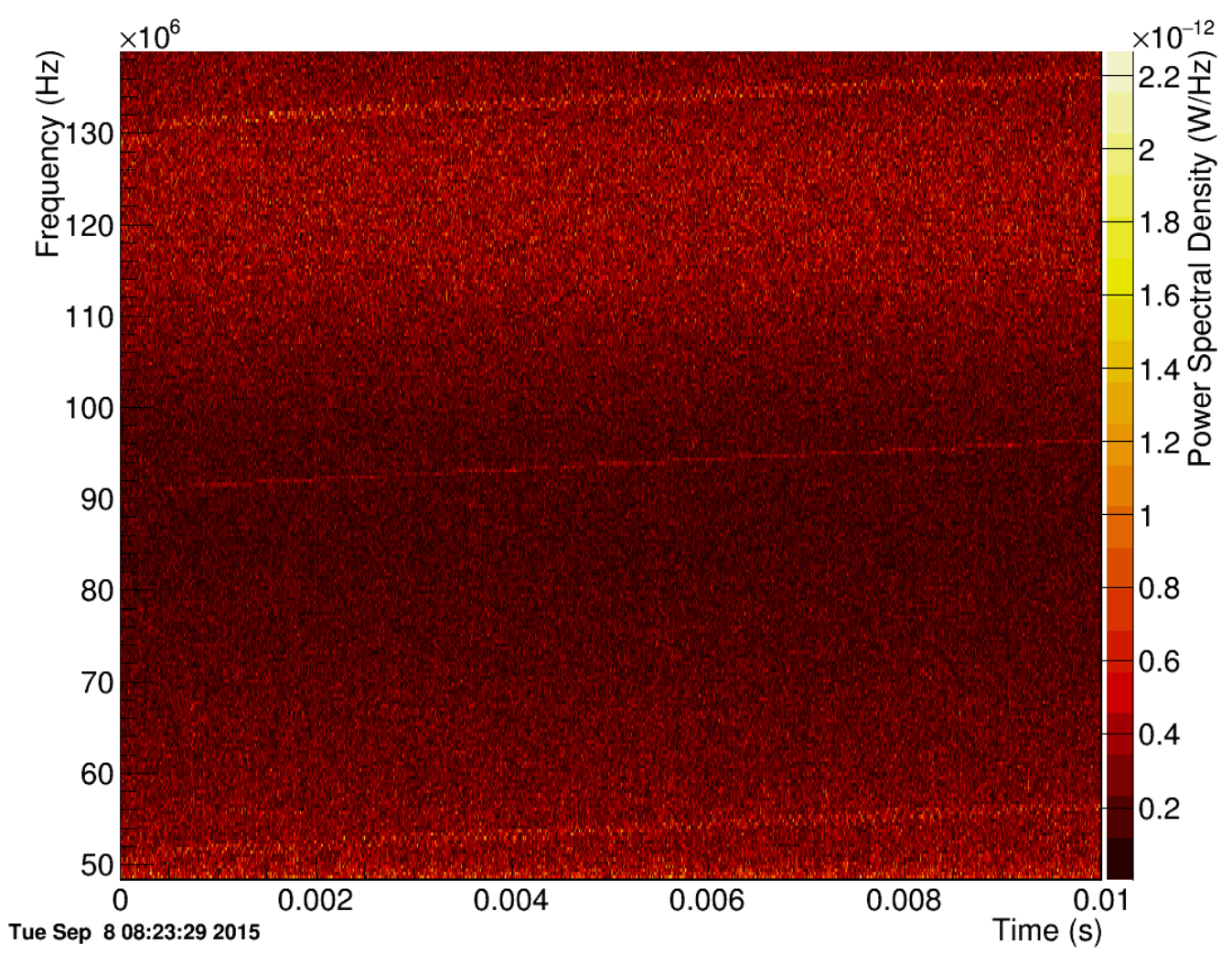

Figure 6: Spectrogram showing an electron event with three tracks: a central track plus two sidebands. The sidebands have a $10 \mathrm{MHz}$ oscillation that may be due to magnetron motion of the electron in an asymmetric trap. 


\section{Measuring the Tritium Spectrum}

\subsection{Phase II: Tritium Demonstrator}

The current phase of the Project 8 experiment is aimed at making the first CRES measurement of the tritium beta-decay spectrum. We switched from a rectangular waveguide to a circular waveguide with a $1 \mathrm{~cm}$ inner diameter to increase the volume of the gas cell. The length of the cell was also increased to $13 \mathrm{~cm}$, and five trapping coils are wrapped around the waveguide. The increased number of trapping coils allows us to explore a variety of trapping configurations. Other improvements include:

- Electron Spin Resonance (ESR) measurements at each trap coil location that allow us to regularly measure the background field along the length of the gas cell;

- A new data acquisition system using the ROACH2 platform [9], which gives us the ability to simultaneously measure three $100 \mathrm{MHz}$-wide frequency windows;

- A cryogenic isolator that was inserted in the RF chain above the gas cell that significantly reduces the detected thermal noise.

All other major components of the experiment, including the superconducting magnet and cryogenic amplifiers, remained the same. We are currently operating the Phase II apparatus with a ${ }^{83 \mathrm{~m}} \mathrm{Kr}$ source as we work to fully understand the data before switching to a tritium source.

\subsection{Phase III: A Scalable Experiment}

The third phase of Project 8 will include the first limit placed on the neutrino mass. We aim to place the same limit as the Mainz and Troitsk experiments, around $2 \mathrm{eV}[2,3]$. In any tritiumendpoint neutrino mass experiment the source volume is a key factor in determining how many electrons near the endpoint will be produced: the fraction of electrons in the last $10 \mathrm{eV}$ of the spectrum is approximately $3 \times 10^{-10}$. The primary goal of Phase III of Project 8 is to create an apparatus that can be easily scaled to larger volumes. In Phases I and II, the gas volume was limited to the inside of a small waveguide. It would difficult to scale such a system to the $\sim 100 \mathrm{~m}^{3}$ volume we need for Phase IV. Instead, we will move to a larger magnet, and a configuration with an array of antennas that sit outside the gas volume as shown in Fig. 7 (left). The free-space radiation from the electrons is detected by a ring array of antennas. This apparatus will observe a gas volume of approximately $200 \mathrm{~cm}^{3}$, and be placed inside of an MRI magnet.

Digital beam-forming will be used to spatially locate electrons within the fiducial volume of the tritium source: by adjusting the relative phases between the individual antenna signals the array can be focused on different locations in the cross section of the source volume. Fig. 7 shows the focus on the cylindrical axis (left) and $4 \mathrm{~cm}$ off-center (right).

\subsection{Phase IV: Atomic Tritium}

Phase IV is the final planned stage of the Project 8 experiment. This apparatus will be capable of measuring the neutrino mass down to $40 \mathrm{meV}$. The most important change from Phase III is the switch to an atomic tritium source. With molecular tritium the ultimate achievable resolution is 

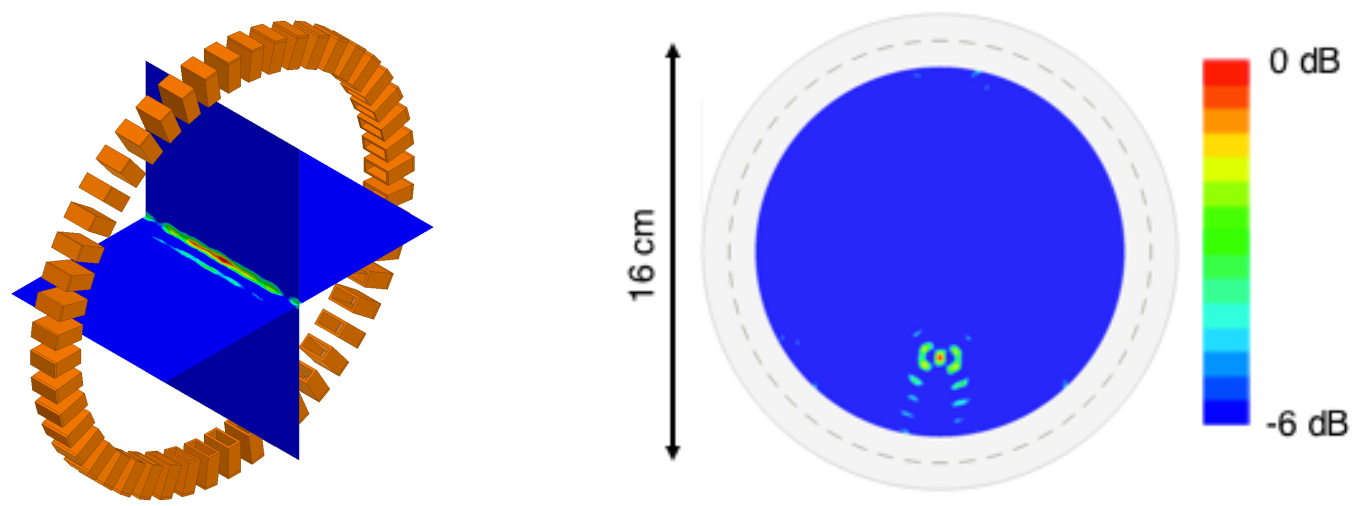

Figure 7: Simulations of a 48-element circular antenna array with the focal region shown in the vertical and horizontal cut planes (left) and the orthogonal projection (right). The focal region is moved by changing the relative phases of the signals from the antennas, and is shown both on the cylindrical axis (left) and $4 \mathrm{~cm}$ below the axis (right).

limited to $100 \mathrm{meV}$ by the vibrational and rotational final states of the ${ }^{3} \mathrm{HeT}^{+}$molecule. By using atomic tritium we can surpass that limitation. The ultimate limit to the resolution for an atomic tritium experiment is estimated to be a result of the magnetic field uncertainty, $\Delta B / B \sim 10^{-7}$. To achieve the statistical precision necessary for a $40 \mathrm{meV}$ measurement we will use a gas volume of roughly $100 \mathrm{~m}^{3}$.

\section{Summary}

The Project 8 experiment is a staged approach to using Cyclotron Radiation Emission Spectroscopy (CRES) to measure the neutrino mass. With Phase I we made the first measurement of single-electron cyclotron radiation, and demonstrated the ability of the CRES technique to resolve the peaks of the ${ }^{83 \mathrm{~m}} \mathrm{Kr}$ spectrum with $\sim 1 \mathrm{eV}$ resolution. In Phase II, currently underway, we will switch to a molecular tritium source and measure the tritium beta-decay energy spectrum. With Phase III we will scale up the volume of the experiment and switch to a new antenna configuration. We will place a limit on the neutrino mass at the level of the Mainz and Troitsk experiments, around $2 \mathrm{eV}$. Phase IV will be the final phase of the experiment, where we will be able to place a limit on the neutrino mass at $\sim 40 \mathrm{meV}$ with an atomic tritium source.

\section{References}

[1] See, for example, E. W. Otten and C. Weinheimer, Rept. Prog. Phys. 71, 086201 (2008).

[2] C. Weinheimer, et al., Phys. Lett. B460, 219 (1999).

[3] Troitsk Collaboration, Phys. Lett. B460, 227 (1999).

[4] C. Patrignani, et al. (Particle Data Group), Chin. Phys. C, 40, 10001 (2016) and 2017 update.

[5] KATRIN Collaboration, arXiv: hep-ex/0109033, (2001).

[6] B. Monreal and J. A. Formaggio, Phys. Rev. D80, 051301 (2009). 
[7] D. M. Asner, et al., Phys. Rev. Lett. 114, 162501 (2015) [arXiv: 1408 . 5362].

[8] A. Ashtari Esfahani, et al., J. Phys. G 44, 5 (2017) [arXiv: 1703 . 02037].

[9] CASPER Collaboration (2017, January 23), ROACH-2 Revision 2, Retrieved from https://casper.berkeley.edu/wiki/ROACH-2_Revision_2. 